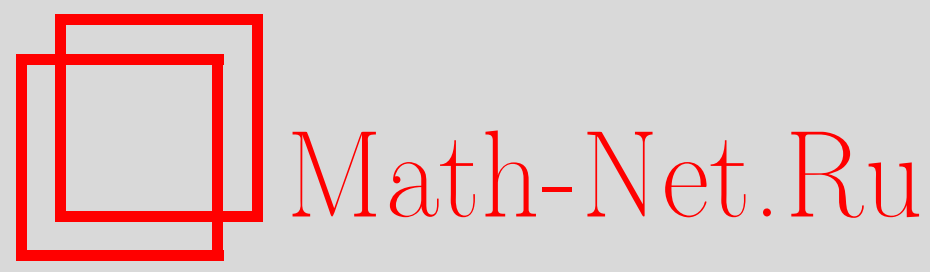

В. А. Емеличев, К. Г. Кузьмин, Об устойчивости векторной комбинаторной задачи с критериями вида MINMIN, Дискрет. матем., 2008, том 20, выпуск 4, 3-7

DOI: https://doi.org/10.4213/dm1022

Использование Общероссийского математического портала Math-Net.Ru подразумевает, что вы прочитали и согласны с пользовательским соглашением http://www.mathnet.ru/rus/agreement

Параметры загрузки:

IP : 54.224 .60 .19

26 апреля 2023 г., 09:19:07 


\title{
Об устойчивости векторной комбинаторной задачи с критериями вида MINMIN
}

\author{
() 2008 г. В. А. Емеличев, К. Г. Кузьмин
}

\begin{abstract}
Рассматривается многокритериальная комбинаторная задача с миниминными критериями. Получено необходимое и одновременно достаточное условие того типа устойчивости задачи, который является дискретным аналогом свойства полунепрерывности сверху по Хаусдорфу многозначного отображения, ставящего в соответствие каждому набору параметров векторного критерия множество Парето задачи.
\end{abstract}

Пусть $E=\left\{e_{1}, e_{2}, \ldots, e_{m}\right\}-$ некоторое множество, $T \subseteq 2^{E} \backslash\{\varnothing\},|T| \geqslant 2, A_{i}-i$-я строка матрицы $A=\left[a_{i j}\right] \in \mathbf{R}^{n \times m}, n \geqslant 1, m \geqslant 2$. Пусть компонентами вектор-функции $f(t, A)=\left(f_{1}\left(t, A_{1}\right), f_{2}\left(t, A_{2}\right), \ldots, f_{n}\left(t, A_{n}\right)\right)$, заданной на множестве (допустимых) решений $T$, являются миниминные критерии вида

$$
f_{i}\left(t, A_{i}\right)=\min _{j \in N(t)} a_{i j} \rightarrow \min _{t \in T}, \quad i \in N_{n}
$$

где $N_{n}=\{1,2, \ldots, n\}, N(t)=\left\{j \in N_{m}: e_{j} \in t\right\}$.

Под векторной ( $n$-критериальной) комбинаторной задачей $Z^{n}(A)$ будем понимать задачу нахождения множества Парето (множества эффективных решений) [1]:

$$
P^{n}(A)=\left\{t \in T: \forall t^{\prime} \in T\left(t \succsim_{A} t^{\prime}\right)\right\}
$$

где $\underset{A}{\succ}-$ как обычно, отрицание бинарного отношения $\underset{A}{\succ}$ :

$$
t \succ_{A} t^{\prime} \Longleftrightarrow f(t, A) \geqslant f\left(t^{\prime}, A\right) \& f(t, A) \neq f\left(t^{\prime}, A\right) .
$$

Отметим, что в схему скалярных $(n=1)$ комбинаторных задач (с линейными, минимаксными и др. критериями) вкладываются многие экстремальные задачи на графах (о коммивояжере, паросочетаниях, остовах и др.), задачи булева программирования и некоторые задачи теории расписаний (см., например, $[2,4,3])$.

В соответствии с определением [5-9] задачу $Z^{n}(A)$ назовем устойчивой (по функционалу), если

$$
\exists \varepsilon>0 \quad \forall B \in \mathscr{B}(\varepsilon) \quad\left(P^{n}(A+B) \subseteq P^{n}(A)\right),
$$

где

$$
\mathscr{B}(\varepsilon)=\left\{B \in \mathbf{R}^{n \times m}:\|B\|<\varepsilon\right\}
$$


- множество возмущающих матриц и

$$
\|B\|=\max \left\{\left|b_{i j}\right|:(i, j) \in N_{n} \times N_{m}\right\} .
$$

Легко понять, что при выполнении равенства $P^{n}(A)=T$ задача $Z^{n}(A)$ устойчива при любой матрице $A \in \mathbf{R}^{n \times m}$. Задачу $Z^{n}(A)$, для которой непусто множество $\bar{P}^{n}(A)=T \backslash P^{n}(A)$, будем называть нетривиальной.

Множество Слейтера (множество слабо эффективных решений) зададим, как обычно, формулой [1]

$$
S l^{n}(A)=\left\{t \in T: \nexists t^{\prime} \in T \forall i \in N_{n}\left(f_{i}\left(t, A_{i}\right)>f_{i}\left(t^{\prime}, A_{i}\right)\right)\right\} .
$$

Очевидно, что $\varnothing \neq P^{n}(A) \subseteq S l^{n}(A)$ при всякой матрице $A \in \mathbf{R}^{n \times m}$.

Известно [6], что совпадение множеств Парето и Слейтера, будучи достаточным условием устойчивости нетривиальной задачи $Z^{n}(A)$ (см. ниже следствие 3 ), не является необходимым. Данная работа посвящена получению критерия устойчивости этой задачи.

Введем обозначения

$$
\begin{array}{rlrl}
V(t, I, A) & =\prod_{i \in I} N_{i}\left(t, A_{i}\right), & & I \subseteq N_{n}, \\
N_{i}\left(t, A_{i}\right) & =\operatorname{Argmin}\left\{a_{i j}: j \in N(t)\right\}, & & i \in N_{n}, \\
P^{n}(t, A) & =\left\{t^{\prime} \in P^{n}(A): f(t, A) \geqslant f\left(t^{\prime}, A\right)\right\}, & & \\
I\left(t, t^{\prime}\right) & =\left\{i \in N_{n}: f_{i}\left(t, A_{i}\right)=f_{i}\left(t^{\prime}, A_{i}\right)\right\} . &
\end{array}
$$

Очевидно, что

$$
f_{i}\left(t, A_{i}\right)=a_{i j} \quad \forall j \in N_{i}\left(t, A_{i}\right),
$$

$I\left(t, t^{\prime}\right) \neq \varnothing$ при любых $t \in S l^{n}(A), t^{\prime} \in P^{n}(t, A)$. Поскольку множество $T$ конечно, то множество Парето $P^{n}(A)$ непусто и, более того, внешне устойчиво [1], то есть $P^{n}(t, A) \neq \varnothing$ при любом решении $t \in T$, причем $t \in P^{n}(t, A)$ тогда и только тогда, когда $t \in P^{n}(A)$.

Через $v_{I}$ будем обозначать проекцию вектора $v \in \mathbf{R}^{n}$ на координатные оси с номерами из множества $I \subseteq N_{n}$. Задачу $Z^{n}(A)$ назовем регулярной, если для всякого решения $t \in S l^{n}(A)$ справедлива формула

$$
\forall v \in V\left(t, N_{n}, A\right) \quad \exists t^{*} \in P^{n}(t, A) \quad\left(v_{I\left(t, t^{*}\right)} \in V\left(t^{*}, I\left(t, t^{*}\right), A\right)\right) .
$$

Теорема 1. Для того чтобы векторная нетривиальная задача $Z^{n}(A), n \geqslant 1$, с частными критериями вида MINMIN была устойчива, необходимо и достаточно, чтобы она была регулярной.

Доказательство. Докажем необходимость. Пусть нетривиальная задача устойчива. Покажем, что для всякого решения $t \in S l^{n}(A)$ выполняется формула (2).

Если $t \in P^{n}(A)$, то ввиду того, что $t \in P^{n}(t, A)$ и $I(t, t)=N_{n}$, для любого вектора $v \in V\left(t, N_{n}, A\right)$ справедливо включение $v_{I(t, t)} \in V(t, I(t, t), A)$. Таким образом, формула (2) верна при $t \in P^{n}(A)$.

Доказательство (2) для любого решения $t \in S l^{n}(A) \backslash P^{n}(A)$, проведем методом от противного. Пусть найдутся $t^{0} \in S l^{n}(A) \backslash P^{n}(A), v^{0}=\left(v_{1}^{0}, v_{2}^{0}, \ldots, v_{n}^{0}\right) \in V\left(t^{0}, N_{n}, A\right)$ такие, что

$$
\forall t \in P^{n}\left(t^{0}, A\right) \quad\left(v_{I\left(t, t^{0}\right)}^{0} \notin V\left(t, I\left(t, t^{0}\right), A\right)\right),
$$


то есть для всякого решения $t \in P^{n}\left(t^{0}, A\right)$, отличного от $t^{0}$, существует индекс $q \in I\left(t, t^{0}\right)$ такой, что $v_{q}^{0} \notin N_{q}\left(t, A_{q}\right)$. Тогда

$$
f_{q}\left(t^{0}, A_{q}\right)=f_{q}\left(t, A_{q}\right), \quad v_{q}^{0} \in N_{q}\left(t^{0}, A_{q}\right) \backslash N_{q}\left(t, A_{q}\right) .
$$

Поэтому, задав элементы возмущающей матрицы $B^{0}=\left[b_{i j}^{0}\right] \in \mathscr{B}(\varepsilon)$ по правилу

$$
b_{i j}^{0}= \begin{cases}-\alpha, & \text { если } i \in N_{n}, j=v_{i}^{0}, \\ 0 & \text { в остальных случаях, }\end{cases}
$$

где $0<\alpha<\varepsilon$, находим, что

$$
f_{q}\left(t^{0}, A_{q}+B_{q}^{0}\right)=f_{q}\left(t^{0}, A_{q}\right)-\alpha<f_{q}\left(t, A_{q}\right)=f_{q}\left(t, A_{q}+B_{q}^{0}\right) .
$$

Итак, для всякого решения $t \in P^{n}\left(t^{0}, A\right)$

$$
t^{0} \underset{A+B^{0}}{\bar{\succ}} t
$$

Пусть $t \in T \backslash P^{n}\left(t^{0}, A\right)$. Найдется такой индекс $s \in N_{n}$, что $f_{s}\left(t^{0}, A_{s}\right)<f_{s}\left(t, A_{s}\right)$. Из этого неравенства, учитывая строение возмущающей матрицы $B^{0}$, получаем соотношения

$$
f_{s}\left(t^{0}, A_{s}+B_{s}^{0}\right)=f_{s}\left(t^{0}, A_{s}\right)-\alpha<f_{s}\left(t, A_{s}\right)-\alpha \leqslant f_{s}\left(t, A_{s}+B_{s}^{0}\right),
$$

которые вновь приводят к (3).

Резюмируя вышесказанное, видим, что $t^{0} \in P^{n}\left(A+B^{0}\right)$. Поэтому, учитывая, что $t^{0} \in \bar{P}^{n}(A)$, получаем:

$$
\forall \varepsilon>0 \quad \exists B^{0} \in \mathscr{B}(\varepsilon) \quad\left(P^{n}\left(A+B^{0}\right) \not P^{n}(A)\right) .
$$

Следовательно, задача $Z^{n}(A)$ не является устойчивой.

Докажем достаточность. Ввиду нетривиальности задачи $Z^{n}(A)$ очевидно существование положительного числа

$$
\alpha=\min \left\{\left|a_{i j}-a_{i^{\prime} j^{\prime}}\right|>0:(i, j) \in N_{n} \times N_{m},\left(i^{\prime}, j^{\prime}\right) \in N_{n} \times N_{m}\right\} .
$$

Пусть $B=\left[b_{i j}\right]$ - произвольная возмущающая матрица из множества $\mathscr{B}(\varepsilon)$, где $\varepsilon=\alpha / 2$, со строками $B_{i}, i \in N_{n}$. Тогда верны включения

$$
N_{i}\left(t, A_{i}+B_{i}\right) \subseteq N_{i}\left(t, A_{i}\right), i \in N_{n}, t \in T,
$$

Отсюда и из (1) вытекают равенства

$$
\begin{aligned}
f_{i}\left(t, A_{i}+B_{i}\right) & =\min _{j \in N_{i}\left(t, A_{i}+B_{i}\right)}\left(a_{i j}+b_{i j}\right)=\min _{j \in N_{i}\left(t, A_{i}\right)}\left(a_{i j}+b_{i j}\right) \\
& =\min _{j \in N_{i}\left(t, A_{i}\right)}\left(f_{i}\left(t, A_{i}\right)+b_{i j}\right) \\
& =f_{i}\left(t, A_{i}\right)+\min _{j \in N_{i}\left(t, A_{i}\right)} b_{i j}, \quad i \in N_{n},
\end{aligned}
$$

справедливые для любого решения $t \in T$.

Далее, полагая $t \in \bar{P}^{n}(A)$, рассмотрим два возможных случая. 
В первом случае $t \in T \backslash S l^{n}(A)$. Тогда в соответствии с определением множества Слейтера существует такое решение $t^{*} \in T$, что для любого индекса $i \in N_{n}$ верно неравенство

$$
f_{i}\left(t, A_{i}\right)>f_{i}\left(t^{*}, A_{i}\right)
$$

а потому согласно (4) - и неравенство $f_{i}\left(t, A_{i}\right)-f_{i}\left(t^{*}, A_{i}\right) \geqslant \alpha$. Это вместе с (6) приводит к соотношениям

$$
\begin{aligned}
f_{i}\left(t, A_{i}+B_{i}\right)-f_{i}\left(t^{*}, A_{i}+B_{i}\right) & =f_{i}\left(t, A_{i}\right)+\min _{j \in N_{i}\left(t, A_{i}\right)} b_{i j}-f_{i}\left(t^{*}, A_{i}\right)-\min _{j \in N_{i}\left(t^{*}, A_{i}\right)} b_{i j} \\
& \geqslant \alpha-2\|B\|>0, \quad i \in N_{n} .
\end{aligned}
$$

Отсюда следует, что $t \underset{A+B}{\succ} t^{*}$.

Во втором случае $t \in S l^{n}(A)$. Пусть $v=\left(v_{1}, v_{2}, \ldots, v_{n}\right) \in V\left(t, N_{n}, A+B\right)$. Тогда согласно (5) выполняется включение $v \in V\left(t, N_{n}, A\right)$. Поэтому в силу регулярности задачи существует решение $t^{*} \in P^{n}(t, A)$ с условием $v_{I\left(t, t^{*}\right)} \in V\left(t^{*}, I\left(t, t^{*}\right), A\right)$, где $I\left(t, t^{*}\right) \neq \varnothing$, то есть $v_{i} \in N_{i}\left(t, A_{i}\right) \cap N_{i}\left(t^{*}, A_{i}\right)$ при любом $i \in I\left(t, t^{*}\right)$. Отсюда с учетом (1) и (6) получаем, что

$$
\begin{aligned}
f_{i}\left(t, A_{i}+B_{i}\right) & =a_{i v_{i}}+b_{i v_{i}}=f_{i}\left(t, A_{i}\right)+b_{i v_{i}} \\
& =f_{i}\left(t^{*}, A_{i}\right)+b_{i v_{i}} \geqslant f_{i}\left(t^{*}, A_{i}\right)+\min _{j \in N_{i}\left(t^{*}, A_{i}\right)} b_{i j} \\
& =f_{i}\left(t^{*}, A_{i}+B_{i}\right), \quad i \in I\left(t, t^{*}\right) .
\end{aligned}
$$

Учитывая включение $t^{*} \in P^{n}(t, A)$, легко видеть, что множество $N_{n} \backslash I\left(t, t^{*}\right)$ непусто и для каждого индекса $i \in N_{n} \backslash I\left(t, t^{*}\right)$ имеет место неравенство (7). Далее, рассуждая аналогично первому случаю, получаем неравенства

$$
f_{i}\left(t, A_{i}+B_{i}\right)>f_{i}\left(t^{*}, A_{i}+B_{i}\right), \quad i \in N_{n} \backslash I\left(t, t^{*}\right) .
$$

Отсюда, вследствие соотношений (8) вновь убеждаемся, что $t \underset{A+B}{\succ} t^{*}$.

Итак, справедлива формула

$$
\exists \varepsilon>0 \quad \forall B \in \mathscr{B}(\varepsilon) \quad \forall t \in \bar{P}^{n}(A) \quad\left(t \in \bar{P}^{n}(A+B)\right) .
$$

Следовательно, задача $Z^{n}(A)$ устойчива.

Теорема 1 доказана.

Следствие 1. Если векторная нетривиальная задача $Z^{n}(A), n \geqslant 1$, устойчива, то

$$
\forall t \in S l^{n}(A) \quad \exists t^{*} \in P^{n}(A) \quad\left(t \cap t^{*} \neq \varnothing\right) .
$$

Доказательство. Предположим, что формула (9) не выполняется, то есть существует такое решение $t^{0} \in S l^{n}(A)$, что для каждого $t \in P^{n}(A)$, а следовательно, и для каждого $t \in P^{n}\left(t^{0}, A\right)$, пересечение $t \cap t^{0}$ пусто. Тогда $N_{i}\left(t, A_{i}\right) \cap N_{i}\left(t^{0}, A_{i}\right)=\varnothing$ для любого индекса $i \in N_{n}$. Поэтому справедлива формула

$$
\forall t \in P^{n}\left(t^{0}, A\right) \quad \forall v \in V\left(t^{0}, N_{n}, A\right) \quad\left(v_{I\left(t, t^{0}\right)} \notin V\left(t, I\left(t, t^{0}\right), A\right)\right),
$$

свидетельствующая о том, что задача $Z^{n}(A)$ не является регулярной, и потому по теореме 1 не может быть устойчивой.

Следствие 1 доказано. 
Следствие 2. Если верна формула

$$
\forall t \in S l^{n}(A) \quad \exists t^{*} \in P^{n}(t, A) \quad \forall i \in I\left(t, t^{*}\right) \quad\left(N_{i}\left(t, A_{i}\right) \subseteq N_{i}\left(t^{*}, A_{i}\right)\right),
$$

то векторная задача $Z^{n}(A), n \geqslant 1$, устойчива.

Доказательство. Действительно, если для всех $i \in I\left(t, t^{*}\right)$ выполняется включение $N_{i}\left(t, A_{i}\right) \subseteq N_{i}\left(t^{*}, A_{i}\right)$, то $V\left(t, I\left(t, t^{*}\right), A\right) \subseteq V\left(t^{*}, I\left(t, t^{*}\right), A\right)$. Поэтому из (10) вытекает формула

$$
\forall t \in S l^{n}(A) \quad \exists t^{*} \in P^{n}(t, A) \quad \forall v \in V\left(t, N_{n}, A\right) \quad\left(v_{I\left(t, t^{*}\right)} \in V\left(t^{*}, I\left(t, t^{*}\right), A\right)\right),
$$

на основании которой заключаем, что для каждого решения $t \in S l^{n}(A)$ имеет место (2), то есть задача $Z^{n}(A)$ регулярна, а потому устойчива в силу теоремы 1 .

Используя следствие 2, нетрудно убедиться в справедливости следующего утверждения.

Следствие 3. Любое из следующих условий является достаточным для устойчивости векторной задачи $Z^{n}(A), n \geqslant 1$ :

(1) $P^{n}(A)=S l^{n}(A)$,

(2) каждая строка матрищьы А состоит из попарно различных элементов.

Поскольку $P^{1}(A)=S l^{1}(A)$, из следствия 3 вытекает следующее утверждение.

Следствие 4. Скалярная задача $Z^{1}(A)$ устойчива при любом векторе $A \in \mathbf{R}^{m}$.

\section{Список литературы}

1. Подиновский В. В., Ногин В. Д., Парето-оптимальные решения многокритериальных задач. Наука, Москва, 1982.

2. Гордеев Э. Н., Леонтьев В. К., Общий подход к исследованию устойчивости решений в задачах дискретной оптимизации. Журнал вычисл. матем. и матем. физики (1996) 36, №1, 66-72.

3. Sotskov Yu. N., Leontev V. K., Gordeev E. N., Some concepts of stability analysis in combinatorial optimization. Discrete Appl. Math. (1995) 58, 169-190.

4. Сотсков Ю. Н., Сотскова Н. Ю., Теория расписаний. Системы с неопределенными числовыми параметрами. ОИПИ НАН Беларуси, Минск, 2004.

5. Сергиенко И. В., Шило В. П., Задачи дискретной оптимизации. Проблемы, методы решения, исследования. Наукова думка, Киев, 2003.

6. Гирлих Э., Ковалев М. М., Кравцов М. К., Стабильность, устойчивость и квазиустойчивость многокритериальной задачи на системе подмножеств. Кибернетика и системный анализ (1999), № 5 111-124.

7. Емеличев В. А., Кравцов М. К., Об устойчивости в траекторных задачах векторной оптимизации. Кибернетика и системный анализ (1995), № 4 137-143.

8. Emelichev V. A., Girlich E., Nikulin Yu. V., Podkopaev D. P., Stability and regularization of vector problems of integer linear programming. Optimization (2002) 51, 645-676.

9. Емеличев В. А., Кричко В. Н., Формула радиуса устойчивости векторной $l_{\infty}$-экстремальной траекторной задачи. Дискретная математика (2004) 16, №1, 14-20.

Статья поступила 9.11.2007. 